This item was submitted to Loughborough's Research Repository by the author.

Items in Figshare are protected by copyright, with all rights reserved, unless otherwise indicated.

\title{
Effects of lasing in a one-dimensional quantum metamaterial
}

PLEASE CITE THE PUBLISHED VERSION

http://dx.doi.org/10.1103/PhysRevB.91.134513

\section{PUBLISHER}

(C) American Physical Society

VERSION

VoR (Version of Record)

\section{PUBLISHER STATEMENT}

This work is made available according to the conditions of the Creative Commons Attribution-NonCommercialNoDerivatives 4.0 International (CC BY-NC-ND 4.0) licence. Full details of this licence are available at: https://creativecommons.org/licenses/by-nc-nd/4.0/

\section{LICENCE}

CC BY-NC-ND 4.0

\section{REPOSITORY RECORD}

Asai, Hidehiro, Sergey Savel'ev, Shiro Kawabata, and Alexandre M. Zagoskin. 2019. "Effects of Lasing in a One-dimensional Quantum Metamaterial". figshare. https://hdl.handle.net/2134/17722. 


\title{
Effects of lasing in a one-dimensional quantum metamaterial
}

\author{
Hidehiro Asai, ${ }^{1,2}$ Sergey Savel'ev, ${ }^{2,3}$ Shiro Kawabata, ${ }^{1}$ and Alexandre M. Zagoskin ${ }^{2,3,4}$ \\ ${ }^{1}$ Electronics and Photonics Research Institute, National Institute of Advanced Industrial Science and Technology, \\ Tsukuba, Ibaraki 305-8568, Japan \\ ${ }^{2}$ Department of Physics, Loughborough University, Loughborough LE11 3TU, United Kingdom \\ ${ }^{3}$ Center for Emergent Matter Science, RIKEN, Wako-shi, Saitama 351-0198, Japan \\ ${ }^{4}$ Theoretical Physics and Quantum Technologies Department, Moscow Institute for Steel and Alloys, 119049 Moscow, Russia
}

(Received 6 November 2014; revised manuscript received 6 March 2015; published 23 April 2015)

\begin{abstract}
Electromagnetic pulse propagation in a quantum metamaterial, an artificial, globally quantum coherent optical medium, is numerically simulated. We show that a one-dimensional quantum metamaterial based on superconducting quantum bits, initialized in an easily reachable factorized excited state, demonstrates lasing in the microwave range, accompanied by the chaotization of qubit states and generation of higher harmonics. These effects may provide a tool for characterization and optimization of quantum metamaterial prototypes.
\end{abstract}

DOI: 10.1103/PhysRevB.91.134513

PACS number(s): 81.05.Xj, 78.67.Pt, 74.81.Fa, 42.50.-p

\section{INTRODUCTION}

The rapid development of quantum technologies since 2000 resulted in routine fabrication of solid-state-based artificial quantum structures, such as qubit arrays, quantum annealers, etc. [1]. The achieved levels of control and global quantum coherence of these systems still fall short of the requirements of universal quantum computing, and better theoretical methods of their simulation and assessment are required [2]. Nevertheless, they are already sufficient or nearly sufficient for the realization of less demanding quantum devices, such as quantum metamaterials [3-9]. These are artificial media, which (i) are composed of quantum coherent unit elements with desired (engineered) parameters, (ii) allow at least limited direct control of quantum states of these elements, and (iii) can maintain global coherence for the duration of time, exceeding the traversal time of an electromagnetic signal. Of course, they must also satisfy the standard requirement that the size of a unit cell of the system be much less, in practice at least two times less, than the wavelength of the relevant electromagnetic signal, so that they can be treated as a "medium." The totality of points (i)-(iii) makes a quantum metamaterial a qualitatively different system, with a number of unusual properties and applications. In particular, a bifocal superlens [4] and quantum phase-sensitive antennas [8] were predicted.

Superconducting technology provides arguably the best scalability for multiqubit systems [1,10-16], and much attention is therefore concentrated on superconductor-based quantum metamaterials. Experimental demonstration of a wide range of quantum-optical effects in a single superconducting flux qubit interacting with the electromagnetic field in a onedimensional waveguide [17-19], in quantitative agreement with theoretical models, confirmed that fabrication and investigation of superconducting quantum metamaterials is well within the scope of the existing experimental techniques. The first one-dimensional superconducting quantum metamaterial prototype was recently realized [20].

If the system is initialized in one of its macroscopic excited states, one can expect lasing. Lasing in a single artificial atom (superconducting charge qubit) was observed [21] and treated theoretically $[22,23]$.
Here we consider an extended system composed of a large number of qubits placed in a one-dimensional superconducting waveguide (i.e., a system similar to circuit QED). Direct qubitqubit coupling is absent. In this one-dimensional (1D) quantum metamaterial the lasing, a coherent transition of qubits to the lower-energy state triggered by an initial field pulse traveling through the system, is associated with correlated dynamics of qubit states due to the induced qubit-qubit coupling via their interaction with the electromagnetic field. This dynamics is manifested in two peculiar phenomena, which accompany the signal amplification: appearance of higher harmonics of the electromagnetic field and chaotization of qubit dynamics. In circuit QED devices [24,25] the dimensionless coupling strength between the electromagnetic field and the active elements (artificial atoms) is greatly increased compared to the conventional quantum optics $\left(\sim 10^{-2}\right.$ vs $\sim 10^{-7}$; see [6], Table 4.1, p. 189). This is due to not only the greater dipolar moment of the qubit compared to an atom but also mainly the restricted geometry of the electromagnetic field: the field is confined by the superconducting strips to a narrow 1D space under the strips containing qubits (Fig. 1), leading to an increased field energy density.

The spectrum of the electromagnetic field propagating through our system is that of the Josephson plasma waves, which have the well-known classical dispersion law $\omega^{2}=(1+$ $\left.\lambda_{J}^{2} k^{2}\right) \omega_{J}^{2}$. Here the minimum Josephson plasma frequency in the system is $\omega_{J}$, and the Josephson penetration depth is $\lambda_{J}$. The frequency $\omega_{J}$ depends on the quantum state of the qubits embedded in the quantum metamaterial, and thus, the plasma wave propagation is strongly affected by the spatial variation of qubit quantum states. In the continuous limit, when the field wavelength greatly exceeds the unit cell size, this can be seen as the effective dielectric constant $\epsilon_{\text {eff }}=c^{2} /\left(\omega_{J}^{2} \lambda_{J}^{2}\right)$ influenced by the quantum state of qubits.

The decoherence time of realistic charge qubits and the relaxation times of $1 \mathrm{D}$ superconducting resonators and transmission lines significantly exceed the characteristic times of energy transfer between qubits and the field already in the case of weak qubit-field coupling, so that decoherence and leakage can be neglected $[3,26]$. The accuracy of this approximation increases in our current case of strong qubit-field coupling. On the flip side, now the perturbative approach of Refs. [3,26] 


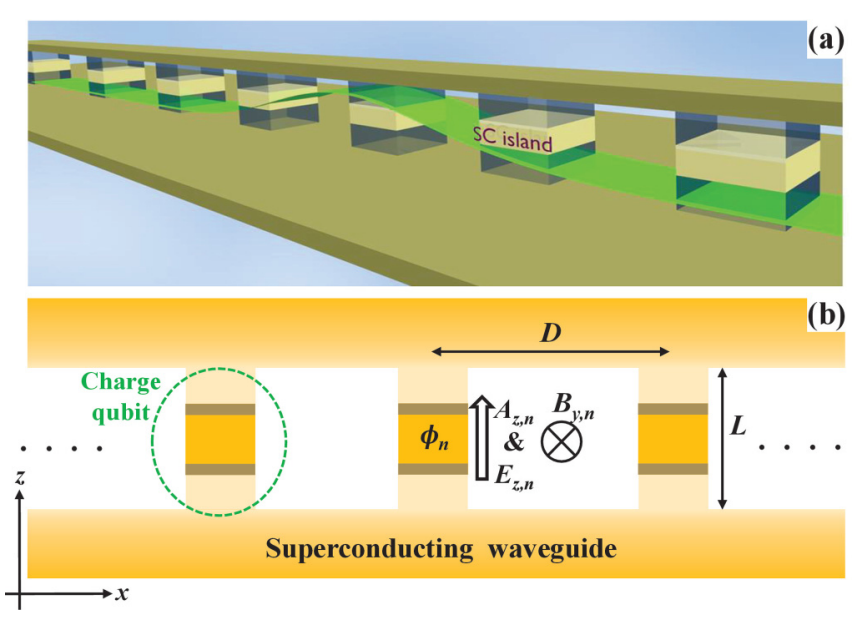

FIG. 1. (Color online) (a) One-dimensional (1D) quantum metamaterial based on charge qubits placed in a waveguide formed by two bulk superconductors. The charge qubit is formed by a superconducting island, separated from the bulk superconductors by identical Josephson junctions, and plays the role of artificial atoms. The green wave represents distribution of the electromagnetic wave in the metamaterial. The control circuitry of qubits (gate electrodes) is not shown. (b) The cross-sectional view of the $1 \mathrm{D}$ quantum metamaterial. The superconducting waveguides are separated by the distance $D$. The charge qubits align at regular intervals $L . \phi_{n}$ is the superconducting phase of the $n$th qubit island. $A_{z, n}, B_{y, n}$, and $E_{z, n}$ are the vector potential, the magnetic field, and the electric field of the $n$th unit cell, respectively.

can no longer be applied. Therefore, we rely on the numerical solution of the equations describing the pulse propagation in the system.

As in $[3,27]$, we treat the electromagnetic field classically, which is the standard approximation in treating atom-field interactions for large field amplitudes (see, e.g., [28]). We also approximate the quantum state of the quantum metamaterial by a product of single-qubit wave functions. The two approximations are consistent. In our model there is no direct qubit-qubit coupling, and the indirect interactions through a classical field do not lead to entanglement (see, e.g.,[29]). The effects of qubit-qubit entanglement for the system with direct qubit coupling and electromagnetic field quantization for weak electromagnetic field (one or two electromagnetic quanta per wavelength) in the system must be investigated in parallel and are beyond the scope of this paper.

As the result of the latter approximation, the complexity of states available to the system is drastically reduced. Indeed, we cannot consider, e.g., quantum birefringence, i.e., the system being in a superposition of states $|000 \cdots 0\rangle$ and $|111 \cdots 1\rangle$ (which correspond to different refractive indices). Nevertheless the approximation is adequate for the description of lasing. This is fortunate since such factorized states are the most robust of all quantum coherent states of a macroscopic system, being, in a sense, the closest to classical ones [30,31]. Their decoherence time is limited by the decoherence time of a single qubit and can therefore greatly exceed the period of field oscillation. [In the continuum limit $[3,6]$ such states are described by a two-component macroscopic wave function $\hat{\Psi}(x)$, formally analogous to superconducting order parameter
$\Delta(x)$. The crucial distinction between them comes from the fact that the former is transitional and eventually disappears, while the latter is an equilibrium quantity, the nonzero value of which is supported and protected from destruction by thermal fluctuations by the interactions in the system.]

\section{MODEL}

We do not expect that the character of signal propagation in a quantum metamaterial will qualitatively depend on whether it is built of charge or flux qubits [6] (or, for that matter, any other kind of superconducting qubit). We will therefore consider here a quantum metamaterial based on charge qubits [3,27] (Fig. 1). Note that the proof-of-principle lasing experiment [21] used a single charge qubit, while the demonstration of quantum optical effects in a 1D transmission line geometry [17] was achieved on a single flux qubit.

The separation $D$ between the superconducting waveguides and the period $L$ of the structure is much smaller than the wavelength of the electromagnetic field mode in the waveguide. This allows us to neglect the variation of the electric field and vector potential within each cell and express the (classical) magnetic and electric field in the $n$th cell as

$$
\begin{gathered}
B_{y, n}=\frac{A_{z, n+1}-A_{z, n}}{L}, \\
E_{z, n}=\frac{1}{2} \frac{V_{z, n}^{u}-V_{z, n}^{l}}{D} \\
=\frac{1}{2 D}\left[\frac{\hbar}{2 e} \frac{d}{d t}\left(0-\phi_{n}-\frac{2 \pi}{\Phi_{0}} \int A_{z, n} d z\right)\right. \\
\left.+\frac{\hbar}{2 e} \frac{d}{d t}\left(\phi_{n}-0-\frac{2 \pi}{\Phi_{0}} \int A_{z, n} d z\right)\right] \\
=-\frac{h}{2 e \Phi_{0}} \frac{d A_{z, n}}{d t},
\end{gathered}
$$

where $\phi_{n}$ is the superconducting phase of the $n$th qubit island and $V_{z, n, u}$ and $V_{z, n, l}$ are the voltages on the upper and lower Josephson junctions of this qubit, respectively. The energy of the system can thus be written as the sum over unit cells (we assume that all qubits are identical), $\mathcal{E}_{\text {tot }}=\sum_{n} \mathcal{E}_{n}$, where

$$
\begin{aligned}
\mathcal{E}_{n}= & \frac{\hbar^{2} C}{8 e^{2}}\left[\left(\frac{d \phi_{n}}{d t}+\frac{\pi D}{\Phi_{0}} \frac{d A_{z, n}}{d t}\right)^{2}+\left(\frac{d \phi_{n}}{d t}-\frac{\pi D}{\Phi_{0}} \frac{d A_{z, n}}{d t}\right)^{2}\right] \\
& -E_{J}\left[\cos \left(\phi_{n}+\frac{\pi D A_{z, n}}{\Phi_{0}}\right)+\cos \left(\phi_{n}-\frac{\pi D A_{z, n}}{\Phi_{0}}\right)\right] \\
& +\frac{D L}{8 \pi}\left[\frac{A_{z, n+1}-A_{z, n}}{L}\right]^{2} \\
= & \frac{E_{J}}{2 \omega_{J}^{2}}\left[\left(\frac{d \phi_{n}}{d t}\right)^{2}+\left(\frac{\pi D}{\Phi_{0}} \frac{d A_{z, n}}{d t}\right)^{2}\right] \\
& -2 E_{J} \cos \phi_{n} \cos \frac{\pi D A_{z, n}}{\Phi_{0}}+\frac{D L}{8 \pi}\left[\frac{A_{z, n+1}-A_{z, n}}{L}\right]^{2} .
\end{aligned}
$$

In Eq. (3) the first term is the electrostatic energy, the second is the Josephson energy, and the last one is the magnetic field 
energy. Further, $E_{J}=I_{c} \Phi_{0} / 2 \pi c$ is the Josephson energy, $\omega_{J}^{2}=2 e I_{c} / \hbar C$ is the Josephson plasma frequency, and $I_{c}$ and $C$ are the critical current and the capacitance of the Josephson junctions, respectively. The natural length unit is now $\lambda=c / \omega_{J}$. In the following we will use the dimensionless quantities $l=L / \lambda$ (unit cell length), $a_{z, n}=\pi D A_{z, n} / \Phi_{0}$ (vector potential), $e_{z, n}=\pi D \lambda E_{z, n} / \Phi_{0}$ (electric field), $b_{y, n}=$ $\pi D \lambda B_{y, n} / \Phi_{0}$ (magnetic field), $\tau=\omega_{J} t$ (time), and $E_{n}=$ $\mathcal{E}_{n} / E_{J}$ (energy). This allows us to rewrite (3) as

$$
\begin{aligned}
E_{\mathrm{tot}} & =\sum_{n} E_{n} \equiv \sum_{n}\left\{E_{n}^{\mathrm{qb}}\left(\phi_{n}, a_{z, n}\right)+E_{n}^{\mathrm{field}}\left(a_{z, n}\right)\right\}, \\
E_{n}^{\mathrm{qb}}\left(\phi_{n}, a_{z, n}\right) & =\left(\frac{d \phi_{n}}{d \tau}\right)^{2}-2 \cos \phi_{n} \cos a_{z, n}, \\
E_{n}^{\mathrm{field}}\left(a_{z, n}\right) & =\beta^{2}\left(\frac{a_{z, n+1}-a_{z, n}}{l}\right)^{2}+\left(\frac{d a_{z, n}}{d \tau}\right)^{2},
\end{aligned}
$$

where the dimensionless parameter

$$
\beta=\frac{L \Phi_{0}^{2}}{8 \pi^{3} D \lambda^{2} E_{J}}
$$

characterizes the speed of light in the metamaterial.

The quantization of (4) is straightforward: the qubit energy $E_{n}^{\mathrm{qb}}$ is replaced by the qubit Hamiltonian $\mathcal{H}_{n}^{\mathrm{qb}}$. In order to do so, we note that the time derivative of the qubit phase in (4) is related to the charge on the island via

$$
Q_{n}=-2 e N_{n}=C V_{z, n}^{u}-C V_{z, n}^{l}=-\frac{\hbar C}{e} \frac{d \phi_{n}}{d t},
$$

so that the quantization is achieved by the substitution [6]

$$
\frac{d \phi_{n}}{d t}=\frac{2 e^{2}}{\hbar C} N_{n} \rightarrow \frac{2 e^{2}}{\hbar C} \hat{N}_{n}=\frac{2 e^{2}}{\hbar C} \frac{1}{i} \frac{\partial}{\partial \phi_{n}} .
$$

[The action of a gate potential $V_{g}$, one method of controlling the qubit state, can be taken into account by replacing the righthand side of (6) with $\frac{2 e^{2}}{\hbar C}\left(\hat{N}_{n}-n^{*}\right)$, where $n^{*}=C_{g} V_{g} / 2 e$.] Now the $n$th qubit's Hamiltonian takes the form

$$
\begin{aligned}
\mathcal{H}_{n}^{\mathrm{qb}}\left(\phi_{n}, a_{z, n}\right) & =-\frac{e^{2}}{C} \frac{\partial^{2}}{\partial \phi_{n}^{2}}-2 E_{J} \cos \phi_{n} \cos a_{z, n} \\
& =\mathcal{H}_{n, 0}^{\mathrm{qb}}\left(\phi_{n}\right)+\mathcal{H}_{n, \text { int }}^{\mathrm{qb}}\left(\phi_{n}, a_{z, n}\right),
\end{aligned}
$$

where we have split it into the unperturbed part,

$$
\mathcal{H}_{n, 0}^{\mathrm{qb}}\left(\phi_{n}\right)=-\frac{e^{2}}{C} \frac{\partial^{2}}{\partial \phi_{n}^{2}}-2 E_{J} \cos \phi_{n},
$$

and the field-dependent perturbation,

$$
\mathcal{H}_{n, \text { int }}^{\mathrm{qb}}\left(\phi_{n}, a_{z, n}\right)=2 E_{J} \cos \phi_{n}\left(1-\cos a_{z, n}\right) .
$$

For the above-mentioned reasons, we limit our considerations to the factorized quantum states of the system,

$$
|\Psi\rangle=\bigotimes_{n}\left|\Psi_{n}\right\rangle=\bigotimes_{n}\left(C_{0}^{n}|0\rangle+C_{1}^{n}|1\rangle\right)
$$

(we do not explicitly label by $n$ the states of the $n$th qubit, $|0\rangle$ and $|1\rangle$, since they always appear accompanied by the $C^{n}$ 's). Therefore, we can solve for each unit cell separately, regarding the vector potential as a classical parameter. Since the states $|0\rangle$ and $|1\rangle$ of a charge qubit differ by one Cooper pair, the only nonzero matrix elements of the operator $\cos \phi_{n}$ in the basis $\{|0\rangle,|1\rangle\}$ are $\left\langle 0\left|\cos \phi_{n}\right| 1\right\rangle=\left\langle 1\left|\cos \phi_{n}\right| 0\right\rangle=1[6]$. Therefore, for the coefficients $C_{0,1}^{n}$ in the Schrödinger representation we find

$$
\begin{aligned}
i \frac{d}{d \tau} C_{0}^{n} & =\varsigma\left(1-\cos a_{z, n}\right) C_{1}^{n} \exp [-i \varsigma \epsilon \tau], \\
i \frac{d}{d \tau} C_{1}^{n} & =\varsigma\left(1-\cos a_{z, n}\right) C_{0}^{n} \exp [i \varsigma \epsilon \tau] .
\end{aligned}
$$

Here $\epsilon=\left(\mathcal{E}_{1}-\mathcal{E}_{0}\right) / E_{J}$ is the dimensionless qubit excitation energy, and $\varsigma=E_{J} / \hbar \omega_{J}$.

Now following the general approach of $[3,6]$, we write the Hamilton function $\langle H\rangle$ for the classical variable $a_{z, n}$ by replacing the qubit energy $E_{n}^{q b}$ in expression (4) for the total energy of the system with its quantum expectation values, i.e., with the matrix elements,

$$
E_{n}^{\mathrm{qb}} \rightarrow\left\langle\Psi\left|\hat{H}_{n}^{q b}\left(\phi_{n}, a_{z, n}\right)\right| \Psi\right\rangle
$$

(here $\hat{H}=\mathcal{H} / E_{J}$ ). The canonical momentum $\Pi_{n}=2 \frac{\partial a_{z, n}}{\partial \tau}$. The vector potential then satisfies the Hamilton equations

$$
\begin{aligned}
\frac{\partial}{\partial \tau} \Pi_{n} & =2 \frac{\partial^{2} a_{z, n}}{\partial \tau^{2}}=-\frac{\partial\langle H\rangle}{\partial a_{z, n}} \\
& =-2 V_{n} \sin a_{z, n}+2 \beta^{2} \frac{a_{z, n+1}-2 a_{z, n}+a_{z, n-1}}{l^{2}} .
\end{aligned}
$$

Here

$$
V_{n}=C_{0}^{n *} C_{1}^{n} e^{-i \varsigma \epsilon \tau}+C_{0}^{n} C_{1}^{n *} e^{i \varsigma \epsilon \tau} .
$$

Within the assumptions we made, Eqs. (11) and (13) fully describe the evolution of the system. In the continuous limit, $l \rightarrow 0$, they would reduce to the wave equation for the vector potential coupled to the two-component field $\Psi_{0,1}(x, \tau)$, supplemented by the equations for $\Psi_{0,1}(x, \tau)$.

For a better efficiency of numerics, Eqs. (11) are split into real and imaginary parts and solved using the fourth-order Runge-Kutta method. Equation (13) for the vector potential is rewritten in terms of dimensionless electric and magnetic fields as

$$
\begin{aligned}
\frac{d e_{z, n}}{d \tau} & =\beta^{2} \frac{b_{y, n}-b_{y, n-1}}{l}+V_{n} \sin a_{z, n}, \\
b_{y, n} & =-\frac{a_{z, n+1}-a_{z, n}}{l}, \quad e_{z, n}=-\frac{d a_{z, n}}{d \tau}, \\
\frac{d b_{y, n}}{d \tau} & =-\frac{e_{z, n+1}-e_{z, n}}{l},
\end{aligned}
$$

and the resulting system is solved using the finite-difference time-domain method.

\section{RESULTS AND CONCLUSIONS}

We consider a quantum metamaterial containing $N=1200$ unit cells, with periodic boundary conditions:

$$
a_{z, 0}=a_{z, N-1}, \quad a_{z, 1}=a_{z, N} .
$$

We chose the following values for the dimensionless parameters for the metamaterial: $\varsigma=1, \epsilon=2 \pi, \beta=1, l=0.05$. Its initial state is fully excited: $C_{0}^{n}(0)=0 ; C_{1}^{n}(0)=1$ (that 


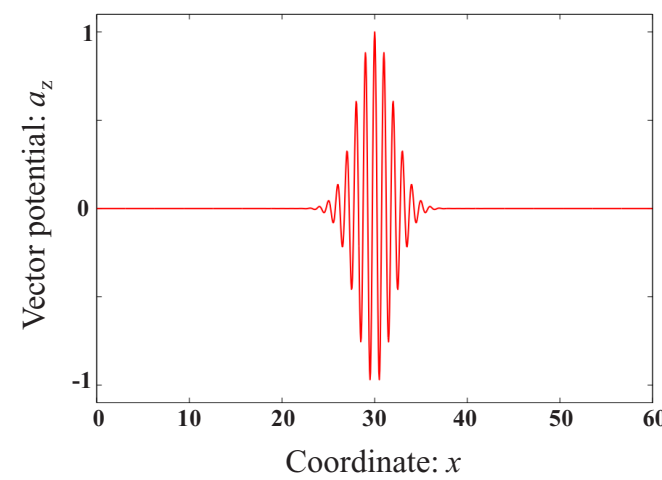

(a)

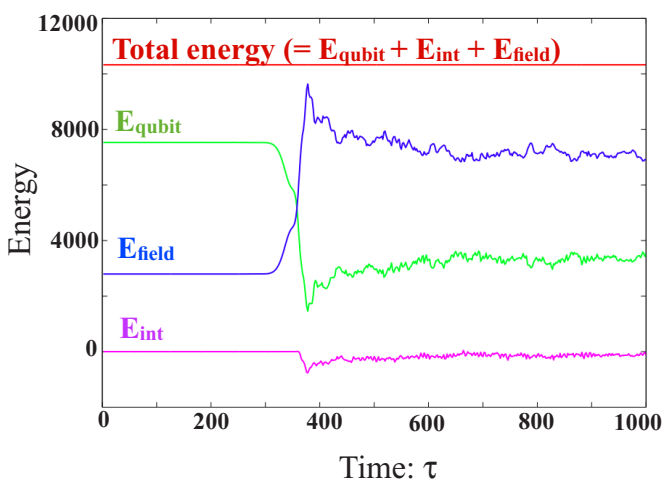

(b)

FIG. 2. (Color online) (a) The initial distribution of vector potential $a_{z}$ in a unit cell (Gaussian pulse). (b) Time evolution of energy in the system. Bottom to top: field-qubit interaction energy $E_{\text {int }}$ (mauve), electromagnetic field energy $E_{\text {field }}$ (blue), qubit energy $E_{\text {qubit }}$ (green), and total energy $E_{\text {total }}($ red $)$.

is, a $100 \%$ population inversion). The interaction of this medium with the electromagnetic field produces a coherent amplification of the initial pulse. Thus, the system is a single-pulse laser.

The initial state of the electromagnetic field is a Gaussian pulse,

$$
\begin{aligned}
a_{z, n}(\tau=0)= & P \exp \left[-\frac{\left(x_{n}+\beta \tau\right)^{2}}{2 q^{2}}\right] \\
& \times \cos \left(k_{g}\left(x_{n}-x_{P}\right)-\omega_{g} \tau\right),
\end{aligned}
$$

where $\omega_{g}=k_{g} \beta$, and we choose $P=1, q=2$, and $\omega_{g}=$ $\epsilon=2 \pi . x_{n}=n l$ is the coordinate of the $n$th qubit along the $x$ direction. The initial field amplitude corresponds to $\sim 7$ photons per wavelength, which then increases to $\sim 26$ photons per wavelength. This makes our classical approximation for the field marginally justified. We set the center of the initial pulse $x_{P}$ at the 600th qubit, that is, $x_{P}=x_{600}=600 l=30$. Figure 2(a) shows the initial distribution of $a_{z, n}(0)$ in the whole system $\left(0 \leqslant n \leqslant 1200,0 \leqslant x_{n} \leqslant 60\right)$. With the chosen parameters, the wave packet traverses the system within $\tau_{0}=60$. Our use of cyclic boundary conditions allows us to investigate the system's behavior on longer times in the regime analogous to the generation regime in a laser with a positive feedback loop.

First, we reassure ourselves that lasing actually occurs from the change of the component of the system energy. Figure 2(b) shows the time evolution of the respective energies; the qubit energy $E_{\text {qubit }}=\sum_{n}\left\langle\Psi_{n}\left|H_{n, 0}^{\mathrm{qb}}\right| \Psi_{n}\right\rangle$, the field-qubit interaction energy $E_{\text {int }}=\sum_{n}\left\langle\Psi_{n}\left|H_{n \text {,int }}^{\mathrm{qb}}\right| \Psi_{n}\right\rangle$, the electromagnetic field energy $E_{\text {field }}=\sum_{n} E_{n}^{\text {field }}$, and the total energy $E_{\text {total }}=E_{\text {qubit }}+E_{\text {int }}+E_{\text {field }}$. In Fig. 2(b), we see that, indeed, at $\tau \sim 300$ the energy of the excited qubits starts pumping energy into the field, and eventually, the field and the qubits almost exactly exchange their energies. The total energy of the system, of course, remains constant. When looking at the electric field distribution and the quantum state of qubits as a function of $\tau$ (Fig. 3), we see that at $\tau=320$ the field absorbs some of the energy of the qubits in its vicinity. As can be seen from these figures, the correlated dynamics of qubit states occurs immediately after the lasing starts $(\tau=300-360)$. This more or less ordered behavior of the quantum metamaterial wave function after several rounds is replaced by a chaotic dependence $\left|C_{1}^{n}\right|$ on the coordinate. By that time the energies of the field and qubits stabilize. It is worth noting that the lasing does not occur immediately after the electromagnetic pulse enters the metamaterial. A delay of lasing is due to the fact that the initial state is a metastable state of our metamaterial, and it takes some time to destabilize qubits by their interaction with field modes.

The numerical calculations established one more interesting feature: the moment when lasing starts depends on the initial field amplitude (see Fig. 4). In Fig. 4, which shows the time evolution of the qubit energy $E_{\text {qubit }}$ for various field amplitudes ( $P=0.02-1.5)$, we see that the moment of lasing becomes early as the amplitude increases. Provisionally, we attribute this to the rigidity of the qubit system, produced by the effective qubit-qubit interaction through the field mode. A stronger qubit-qubit coupling should lead to more correlated behavior of the qubits and should increase the amplitude of the stimulated emission from the quantum metamaterial.

So far we have considered the exact resonance, $\epsilon=\omega_{g}$ [32]. The appearance of a signal at a higher harmonic and subharmonic (see Fig. 5 for the frequency spectrum of the electric field before and after lasing) indicates that lasing should occur as well at other relations between $\epsilon$ and $\omega_{g}$. Indeed, e.g., at $\epsilon=2 \omega_{g}=4 \pi$, we see a clear case of parametric lasing; in this particular case it is driven by two-photon processes. Figure 6 shows the frequency spectrum of the electric field for the case of $\epsilon=2 \omega_{g}=4 \pi$. (Note that other calculation parameters are the same as in the aforementioned calculations.) In this case, lasing immediately occurs after the pulse starts to propagate, and the component of the subharmonic frequency $\frac{\epsilon}{2}$ increases as shown in Fig. 6. Here, as before, after some transition period the qubit and field subsystems exchange their energies and reach stationary states when the qubit states (not shown) are chaotized.

Finally, let us discuss the effects of the energy leakage and decoherence in qubits, using realistic parameters. In order to examine the leakage effects, we have simulated the pulse propagation, including a phenomenological dissipation term $\sigma e_{z, n}$ in the field equations (15), where $\sigma$ is the effective 

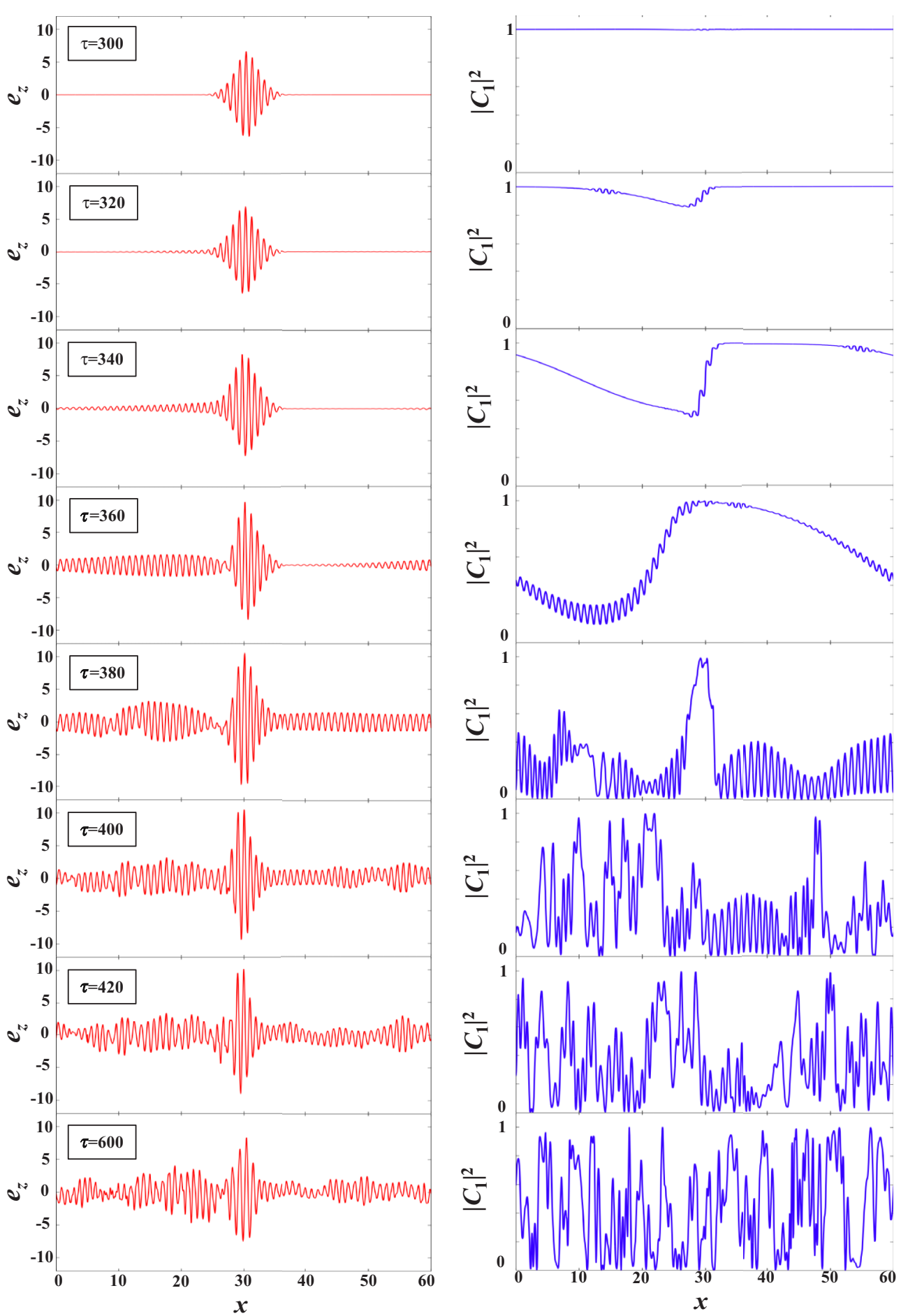

FIG. 3. (Color online) (left) Electric field amplitude and (right) the probability amplitude of the excited state in the metamaterial at $\tau=300,320,340,360,380,400,420$, and 600 (top to bottom). The origin of $x$ is shifted to keep the wave packet at the center of the panel.

conductivity in the qubit. The results are shown in Fig. 7. The time evolution of the field energy $E_{\text {field }}$ is plotted vs time for the pulse with $\epsilon=\omega_{g}, P=1, q=2$, and three different choices of $\sigma, \sigma=1 \times 10^{-4}, 5 \times 10^{-4}, 2.5 \times 10^{-3}$. The crosses indicate the field energy dependence on time in a system with the typical quality factor $10^{4}$ expected in a charge qubit coupled to a strip line [33]. We can see that in the presence of dissipation the lasing begins within $\tau=300-350$ after the initial pulse entering the metamaterial. It occurs even in the case of strong dissipation, $\sigma=5 \times 10^{-4}, 2.5 \times 10^{-3}$, a decay rate higher than that expected in the type of quantum metamaterial considered here. We believe, therefore, that the lasing effect will be experimentally observable. Next, to examine the decoherence effect, we compare the simulation time in our study with typical decoherence time for charge qubits. If we take $E_{J}=10-50 \mu \mathrm{eV}$ and $E_{c}=10 E_{J}$ [34], $\omega_{J}$ becomes $\sim 10^{11}(1 / \mathrm{s})$. In this case, $\tau=10^{4}$ corresponds to the typical decoherence time for charge qubits, $100 \mathrm{~ns}$, and our 


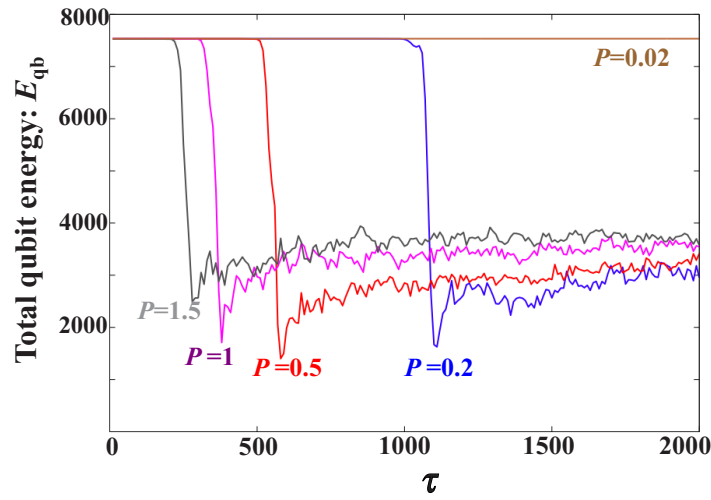

FIG. 4. (Color online) Qubit energy $E_{\text {qubit }}$ as a function of time for various field amplitudes. Right to left: $P=0.05$ (green), 0.2 (blue), 0.5 (pink), 1 (turquoise), 1.5 (gray). At $P=0.02$ (brown) lasing did not start up to $\tau=40000$.

lasing occurs at $\tau \sim 10^{3} \ll 10^{4}$. Thus, our simulations will be justified even in the presence of realistic decoherence.

In conclusion, we have investigated the lasing effects in a one-dimensional quantum metamaterial based on superconducting charge qubits by numerically solving the equations of motion for the system in the presence of a classical electromagnetic pulse. In the case when the signal can propagate repeatedly through the system, the lasing is associated with a correlated behavior of the qubit states, and the electromagnetic pulse and the qubit subsystem exchange their energies.

The onset of lasing strongly depends on the pulse amplitude, and this results from the effective rigidity of the qubit

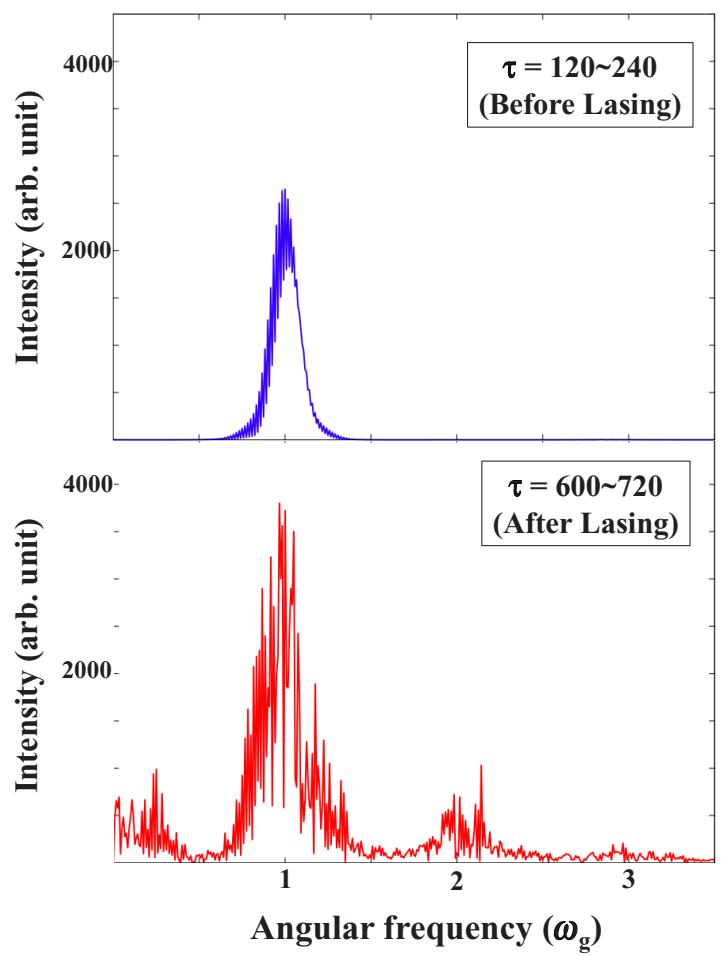

FIG. 5. (Color online) Field frequency spectrum (top) for $\tau=$ 120-240 and (bottom) for $\tau=600-720$. Note the appearance of both higher-harmonic (at $\sim 4 \pi=2 \omega_{g}$ ) and subharmonic peaks after the lasing occurred.

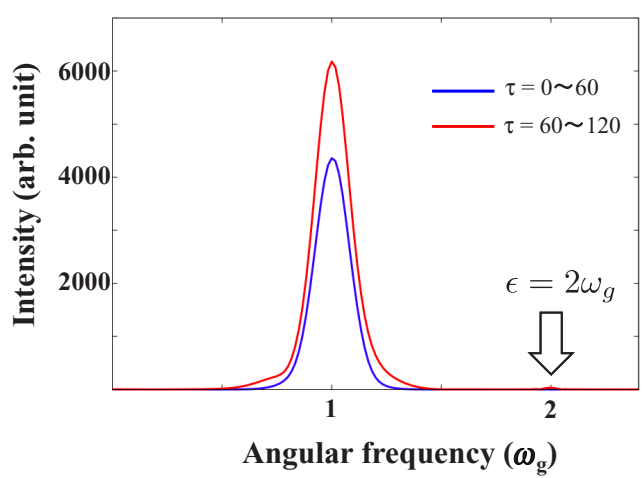

FIG. 6. (Color online) Field frequency spectrum for $\tau=0-60$ (red) and for $\tau=60-120$ (blue) in the case of $\epsilon=2 \omega_{g}=4 \pi$. The subharmonic component $\frac{\epsilon}{2}=\omega_{g}$ increases due to parametric lasing.

subsystem, interacting through the electromagnetic mode. After several rounds of pulse propagation, the system reaches the stationary regime, which is characterized by a chaotic qubit state distribution.

Nonlinear coupling of qubits to the field leads to efficient parametric lasing, e.g., when the pulse frequency is only half of the qubit interlevel distance. The system turns out to have rich physics, within the range of parameters currently available for experimental investigation. Moreover, our quantum metamaterial works as a nonlinear laser medium whose parameters can be artificially controlled and will be a promising candidate for a practical metamaterial.

\section{ACKNOWLEDGMENTS}

H.A. was partially supported by a Grant-in-Aid for JSPS (Japan Society for the Promotion of Science) Fellows, and a Grant-in-Aid for Young Scientists (B) from JSPS (Grant No. 26790062). S.K was partially supported by a Grant-in-Aid for Scientific Research (C) from JSPS (Grant No. 24510146). A.Z. was supported in part by the Ministry of Education and Science of the Russian Federation in the framework of Increase Competitiveness Program of NUST "MISiS" (Grant No. K22014-015).

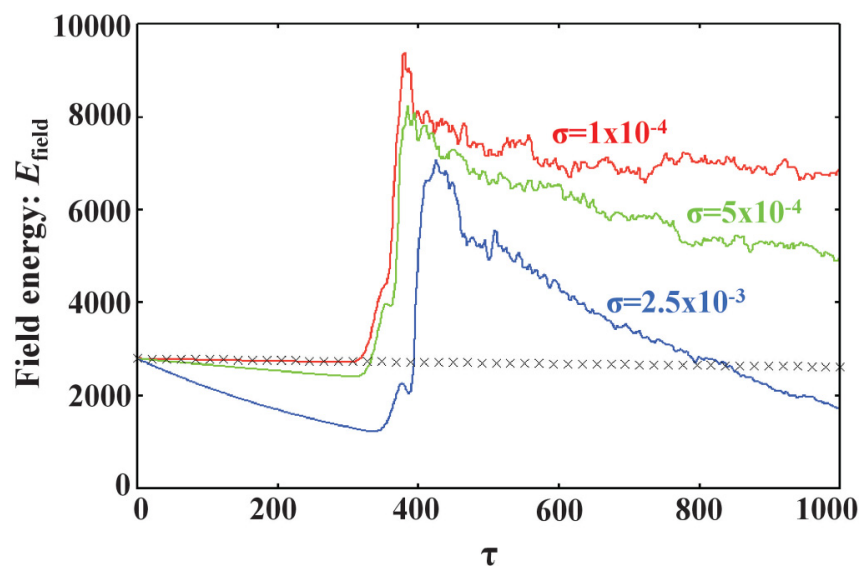

FIG. 7. (Color online) Field energy $E_{\text {field }}$ as a function of time in the presence of dissipation. Left to right: $\sigma=1 \times 10^{-4}$ (red), $5 \times$ $10^{-4}$ (green), $2.5 \times 10^{-3}$ (blue). The cross points indicate the field energy changing with time according to the typical quality factor $10^{4}$. 
[1] Z. L. Xiang, S. Ashhab, J. Q. You, and F. Nori, Rev. Mod. Phys. 85, 623 (2013).

[2] A. M. Zagoskin, E. Ilichev, M. Grajcar, J. J. Betouras, and F. Nori, Front. Phys. 2, 33 (2014).

[3] A. L. Rakhmanov, A. M. Zagoskin, S. Savel'ev, and F. Nori, Phys. Rev. B 77, 144507 (2008).

[4] J. Q. Quach, C. H. Su, A. M. Martin, A. D. Greentree, and L. C. L. Hollenberg, Opt. Express 19, 11018 (2011).

[5] D. Felbacq and M. Antezza, SPIE Newsroom (2012).

[6] A. M. Zagoskin, Quantum Engineering: Theory and Design (Cambridge University Press, Cambridge, 2011).

[7] A. M. Zagoskin, J. Opt. 14, 114011 (2012).

[8] A. M. Zagoskin, R. D. Wilson, M. Everitt, S. Savel'ev, D. R. Gulevich, J. Allen, V. K. Dubrovich, and E. Ilichev, Sci. Rep. 3, 3464 (2013).

[9] M. Biondi, S. Schmidt, G. Blatter, and H. E. Türeci, Phys. Rev. A 89, 025801 (2014).

[10] D. G. Angelakis, M. F. Santos, and S. Bose, Phys. Rev. A 76, 031805 (2007).

[11] J. Q. You and F. Nori, Nature (London) 474, 589 (2011).

[12] A. A. Houck, H. E. Tureci, and J. Koch, Nat. Phys. 8, 292 (2012).

[13] S. Schmidt and J. Koch, Ann. Phys. (Berlin, Ger.) 525, 395 (2013).

[14] I. Carusotto and C. Ciuti, Rev. Mod. Phys. 85, 299 (2013).

[15] J. Jin, D. Rossini, R. Fazio, M. Leib, and M. J. Hartmann, Phys. Rev. Lett. 110, 163605 (2013).

[16] R. Barends et al., Nature (London) 508, 500 (2014).

[17] O. V. Astafiev, A. M. Zagoskin, A. A. Abdumalikov, Jr., Y. A. Pashkin, T. Yamamoto, K. Inomata, Y. Nakamura, and J. S. Tsai, Science 327, 840 (2010).

[18] O. V. Astafiev, A. A. Abdumalikov, Jr., A. M. Zagoskin, Yu. A. Pashkin, Y. Nakamura, and J. S. Tsai, Phys. Rev. Lett. 104, 183603 (2010).
[19] A. A. Abdumalikov, O. V. Astafiev, A. M. Zagoskin, Y. A. Pashkin, Y. Nakamura, and J. S. Tsai, Phys. Rev. Lett. 104, 193601 (2010).

[20] P. Macha, G. Oelsner, J. M. Reiner, M. Marthaler, S. Andre, G. Schoen, U. Huebner, H. G. Meyer, E. Ilichev, and A. V. Ustinov, Nat. Commun. 5, 5146 (2014).

[21] O. V. Astafiev, K. Inomata, A. O. Niskanen, T. Yamamoto, Y. A. Pashkin, Y. Nakamura, and J. S. Tsai, Nature (London) 449, 588 (2007).

[22] S. Ashhab, J. R. Johansson, A. M. Zagoskin, and F. Nori, New J. Phys. 11, 023030 (2009).

[23] S. André, V. Brosco, M. Marthaler, A. Shnirman, and G. Schön, Phys. Scr. T137, 014016 (2009).

[24] A. Blais, R. S. Huang, A. Wallraff, S. M. Girvin, and R. J. Schoelkopf, Phys. Rev. A 69, 062320 (2004).

[25] A. Wallraff, D. I. Schuster, A. Blais, L. Frunzio, R.- S. Huang, J. Majer, S. Kumar, S. M. Girvin, and R. J. Schoelkopf, Nature (London) 431, 162 (2004).

[26] A. M. Zagoskin, E. Ilichev, M. W. McCutcheon, J. F. Young, and F. Nori, Phys. Rev. Lett. 101, 253602 (2008).

[27] A. Shvetsov, A. M. Satanin, F. Nori, S. Savel'ev, and A. M. Zagoskin, Phys. Rev. B 87, 235410 (2013).

[28] D. I. Blokhintsev, Quantum Mechanics (Reidel, Dordrecht, 1964), Chap. XV.

[29] A. Yu. Smirnov and A. M. Zagoskin, arXiv:cond-mat/0207214.

[30] A. Leggett, Found. Phys. 29, 445 (1999).

[31] A. Leggett, J. Supercond. 12, 683 (1999).

[32] We have performed similar simulations for off-resonant pulse frequencies and have found that the lasing phenomena do not change significantly up to $20 \%$ of the off-resonance condition.

[33] J. Gambetta, A. Blais, D. I. Schuster, A. Wallraff, L. Frunzio, J. Majer, M. H. Devoret, S. M. Girvin, and R. J. Schoelkopf, Phys. Rev. A 74, 042318 (2006).

[34] Y. Nakamura, Y. A. Pashkin, and J. S. Tsai, Nature (London) 398, 786 (1999). 